\title{
EXTENDED GAS IN INTERACTING SYSTEMS
}

\author{
F. COMBES \\ Observatoire de Paris, DEMIRM \\ 61 Av. de l'Observatoire, F-75 014 Paris, France
}

\begin{abstract}
HI observations have revealed large gaseous extensions in interacting and merging systems. The interstellar gas is obviously dragged out in tidal tails during an encounter, and the percentage of $\mathrm{HI}$ in the tails increases with the merging stage. However, the opposite is true for the molecular gas, which is observed highly concentrated towards the nuclei of interacting galaxies, amounting to a significant fraction of the dynamical mass. Statistically, there appears to be more gas observed in interacting galaxies than in normal, isolated ones. As N-body simulations show, the gas is driven inwards in the interaction process by the strong gravity torques, before being consumed through star formation in the triggered starbursts. We review here all observations that could bring more knowledge about the state of the gas in the outer parts of galaxies, and about accretion processes. The link with the observations of the Ly $\alpha$ absorbers at low and high redshifts is discussed.
\end{abstract}

\section{Fate of the gas in interacting systems}

One of the main striking features about interacting and merging galaxies, is the presence of large tidal tails of matter dragged out of the galaxies; recent VLA maps have revealed huge HI extensions with respect to the optical systems, as though most of the neutral gas was splashed all around. Yun et al. (1993) have found large quantities of $\mathrm{HI}$ all around the M81/M82/NGC 3077 system, and Hibbard (1995) shows in his thesis an evolving sequence of interacting/merging galaxies, where the $\mathrm{HI}$ extensions are conspicuous. More precisely, the percentage of the total $\mathrm{HI}$ found in the tails/extensions is increasing with the merging stage, from $20 \%$ in the M81 system, to $80 \%$ in the merger remnant NGC 7252. Of course, this can be explained by the tidal potential in the frame of the target growing as $r^{2}$ as a function of the 
target radius, and because matter in the outer parts is less bound to the galaxies. But this gives a wrong idea of the fate of the gas in interacting systems. In fact, with all probability, the gas dragged out remains bound to the system, and will rain back onto the merger remnant, after some billion years. Already, Hibbard (1995) shows that the gas at the bottom of the tails in NGC 7252 is infalling. This progressive infall will take place through phase wrapping, and shells and loops will form.

What is seen in the molecular phase is just the contrary: apparently large $\mathrm{H}_{2}$ concentrations pile up at the galaxy nuclei in interacting systems. Up to $50 \%$ of the dynamical mass could be under the form of molecular hydrogen in merging systems (Scoville et al. 1991). Some ultraluminous infrared galaxies, which are also mergers and starbursts possess $10^{10} M_{\odot}$ of $\mathrm{H}_{2}$ gas, about 10 times more than in their spiral precursors. There exist some hints that there is more gas in interacting galaxies (Braine \& Combes 1993). Although the $\mathrm{H}_{2} / \mathrm{CO}$ conversion ratio is not well known for these peculiar objects, there is evidence for denser gas in these systems, through the HCN/CO ratio (Solomon et al. 1997). In summary, the observations suggest that the HI gas is dragged outwards, while the $\mathrm{H}_{2}$ gas is driven inwards, to be consumed in star formation. In fact these two tracers (HI and $\mathrm{CO}$ ) shed light on two aspects of the same gas component.

The global result is that most of the dissipative component loses angular momentum, and falls inwards towards the nucleus of the merger, although part of it is heated in shocks and pass in the coronal phase (seen in X-rays).

\section{Extension of gas in normal galaxies}

\subsection{SPIRAL GALAXIES}

It is well known that the HI gas sometimes extends much farther than the stars in spiral galactic disks, and they are precious tools to probe the rotation curve and the presence of dark matter. However, large extensions such as in NGC 628, where $R_{\mathrm{HI}}=5 R_{25}$ for instance, are quite exceptional (Kamphuis \& Briggs 1992); only about $10 \%$ have $R_{\mathrm{HI}}$ larger than $2.5 R_{25}$ (Huchtmeier \& Seiradakis 1985). In a sample of about 100 galaxies, chosen to search precisely for extended HI disks, Broeils (1992) did not find many extended gaseous disks. In fact, only regular galaxies were selected, to be able to exploit the rotation curves, and therefore no strongly interacting galaxies are included. The HI diameter, defined at a surface density of 1 $M_{\odot} \mathrm{pc}^{-2}$, is about twice the optical diameter $D_{25}$ (Broeils \& van Woerden 1994). The HI-to-optical-diameter ratio does not depend on morphological type or luminosity (fig. 1), but there is a strong correlation indicating that $M_{\mathrm{HI}} \propto D_{\mathrm{HI}}^{2}$, which means that HI surface density, averaged over the whole HI disk, is constant from galaxy to galaxy, and all over the Hubble sequence 


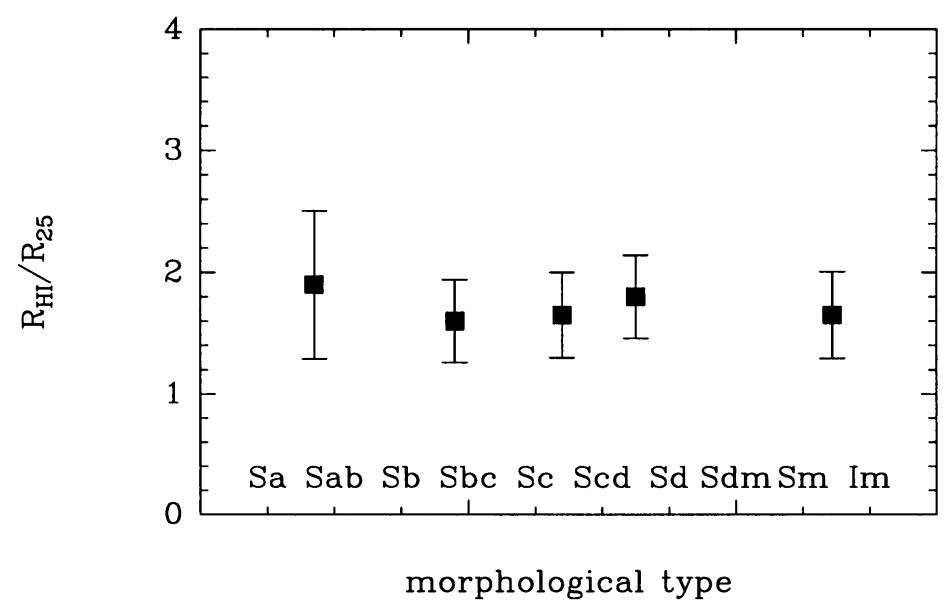

Figure 1. Ratio of HI radius (defined by a surface density of $1 M_{\odot} \mathrm{pc}^{-2}$ ) to optical radius $R_{25}$ as a function of morphological type, from Broeils \& Rhee (1997). The error bars are $1 \sigma$ dispersion.

(Broeils \& Rhee 1997). Besides, there is a strong dependence of the ratio $M_{\mathrm{HI}} / M_{\mathrm{tot}}$ with type, which confirms that the percentage of gas is larger in small late-type galaxies, and decreases all over the Hubble sequence (which is also a mass sequence).

\subsection{DWARF IRREGULAR GALAXIES}

Since dwarf irregular galaxies are particularly rich in HI gas, it was first thought that there might exist big HI envelopes around these objects, or even a large number of isolated gas clouds still waiting to form their first stars. It turned out however that big gas extensions, such as in DDO 154 are very uncommon (e.g. Hoffman et al. 1996), and that the HI-to-opticalradius ratio is very similar in dwarfs and in spirals (cf. fig. 2).

\subsection{THE IONIZATION EDGE OF THE HI DISK}

HI observations have shown that the neutral gas radial distribution is much smoother than that of stars (exponential decrease), or molecular gas as traced by CO (following more or less the blue luminosity). The HI surface density is statistically falling as the dark matter surface density, determined from rotation curves (Bosma 1981, Freeman 1993). This implies an HI column density falling as $1 / r$ at large radii (because the gas layer is flaring linearly with radius, the spatial density in fact is going as $1 / r^{2}$ ). It would 


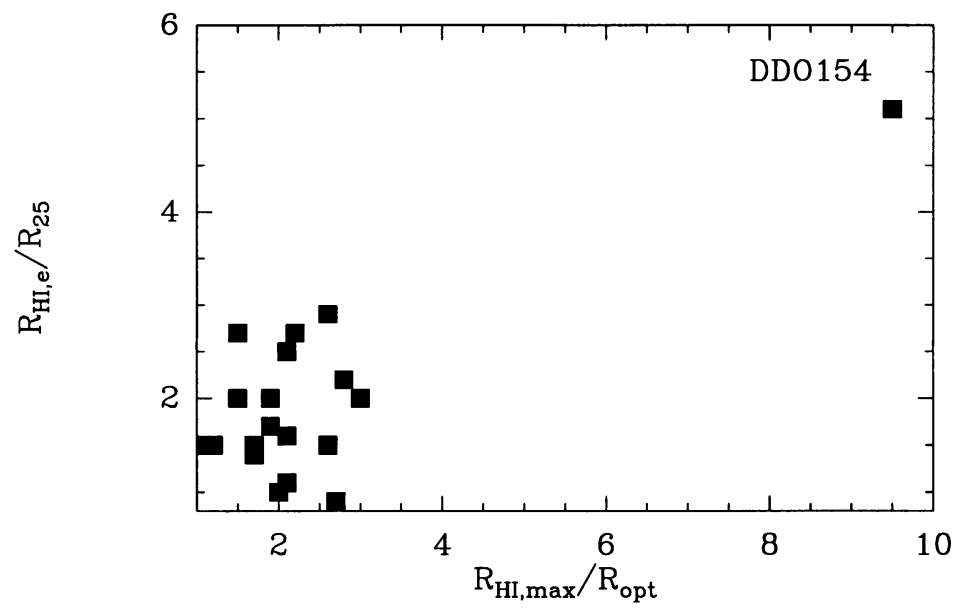

Figure 2. The ratio of maximum HI radius to optical radius, and the ratio of HI radius at 1 /e of the peak flux to the optical isophotal radius, for all resolved dwarfs, studied by Hoffman et al. (1996). Only DDO 154 is outstanding in its HI size.

be interesting to observe the HI until the edges of the neutral disk, to trace its physical condition and also the distribution of the dark matter at large radii, but this is a hard task, due to sensitivity problems. It has been done in a few cases only, in NGC 3198 (van Gorkom 1991) and M33 \& NGC 3344 (Corbelli et al. 1989). The results show a sudden decrease from a column density of $N_{\mathrm{HI}}=2 \cdot 10^{19} \mathrm{~cm}^{-2}$ to $2 \cdot 10^{18} \mathrm{~cm}^{-2}$ in a very short length-scale of 2-4 kpc (of course limited by the spatial resolution of the observations). This sharp edge has been interpreted in terms of the ionization front of the gas disk (Corbelli \& Salpeter 1993): the data can be reproduced if the extragalactic background, essentially from the quasar UV light, provides an ionization rate of $\xi \sim 2 \cdot 10^{-14} \mathrm{~s}^{-1}$, a value corresponding to the study of low-redshift Lyman- $\alpha$ absorption lines (Madau 1992).

The conclusion is that the gas surface density is likely to continue to decrease as $1 / r$ at large distances, but it is difficult to see it. It is even possible that the gas density in the outer parts is highly underestimated, if it is clumpy down to very small scales, and under a cold molecular phase (Pfenniger et al. 1994, Pfenniger \& Combes 1994). Large HI extensions, that are only seen when galaxies are interacting, and molecular gas concentrations, could then reveal the presence of the clumps when they are stirred up by tidal interactions, driven inwards and concentrated. 


\subsection{HOW TO TRACE GAS AT LARGE RADII?}

Gas at large radii is difficult to trace, because of its low average column density, its low temperature, low metallicity $Z$ and dust content ( $Z$ is decreasing exponentially with radius, e.g. Smartt \& Rolleston 1997). Even at any radii, the usual tracers are far from perfect: the HI line can be optically thick (e.g. Burton 1992), CO emission can be absent by lack of excitation for example (Adler et al 1991). Nelson et al. (1996) claim to have detected cold dust at large radii in nearby galaxies through its $100 \mu$ emission; but the very cold dust could be more easily traced at $1.3 \mathrm{~mm}$. Recent continuum maps at this wavelength (which is in the Rayleigh-Jeans domain), are still limited by sensitivity, but they reveal interesting radial distributions. In galaxies where the interstellar medium (ISM) is dominated by the molecular component, like NGC 891 , the $1.3 \mathrm{~mm}$ flux radial distribution is superposable to the $\mathrm{CO}$ one (Guélin et al. 1993), suggesting that the $\mathrm{CO}$ emission is directly proportional to metallicity (like dust emission since $S_{1.3 \mathrm{~mm}}$ is proportional to the dust temperature, the column density of the gas, and the metallicity). On the contrary, in galaxies where the ISM is dominated by the atomic hydrogen, like NGC 4565 , the $1.3 \mathrm{~mm}$ continuum flux follows more the HI emission, which is decreasing more slowly with radius than the $\mathrm{CO}$ one (Neininger et al. 1996), but still the dust emission is falling more rapidly than the HI, because of its $Z$ dependency. In spite of sensitivity and metallicity limitations, dust emission could be one of the best way to trace extended cold gas (e.g. Combes \& Pfenniger 1997).

\subsection{STABILITY OF EXTENDED GASEOUS DISKS}

If extended gaseous disks exist, their gravitational stability raises interesting problems. Since the HI gas at large radii is usually observed lopsided (Richter \& Sancisi 1994), or with multiple spiral arms and large-scale instabilities, it must possess a minimum of self-gravity (Pfenniger et al. 1994). As for small scale instabilities, as well as the vertical equilibrium, constraints can be put on the flattening of the dark matter haloes, which determines its volume density in the plane, as shown in fig. 3 (cf. Olling 1995, 1996, Becquaert \& Combes 1997).

\section{Intergalactic medium}

\subsection{GALAXY CLUSTERS}

Through frequent tidal interactions or harassment (Moore et al. 1996), and ram-pressure stripping (Cayatte et al. 1990), the extended gaseous disks are truncated in rich clusters. Globally, the HI surface density is conserved for spirals in clusters, only their HI-to-optical-radius ratio is smaller (Cayatte et 


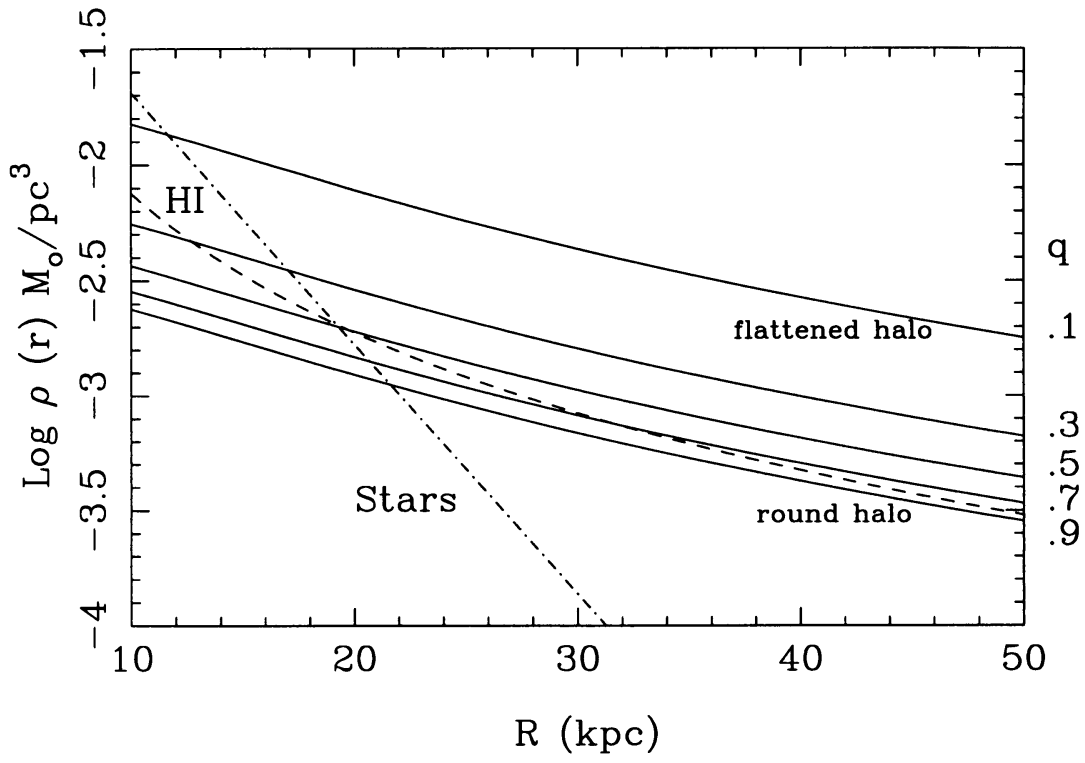

Figure 3. Volume density as a function of radius for the various mass components in a typical galaxy like the Milky Way: exponential stellar disk (dot-dash), flaring HI layer (dash), and isothermal spheroidal halo of flattening q (full lines).

al. 1994), and since their CO content is also normal (Casoli et al. 1996), their central gaseous disk appears not too much perturbed, except for a small number of anemics. The gas coming from the outer parts of galaxies must be now under the form of X-ray emitting hot gas, at the virial temperature of the cluster, and enriched in heavy elements from the galaxies (Renzini 1997).

\subsection{LYMAN- $\alpha$ ABSORBERS}

The intergalactic medium is traced through Lyman- $\alpha$ absorption in front of quasars, especially well sampled at $z=2$ from the ground. Space observations have shown that the absorbers are still present until $z=0$, although with some evolution in number density. The distribution of HI column densities derived from these probes is a continuous power-law (slope $\sim-1.6$ ) up to damped Ly- $\alpha$ values of $10^{20}-10^{21} \mathrm{~cm}^{-2}$, comparable to the outer parts of galaxy disks. Between $z=2$ and $z=3$, most of the baryons are in the Lyman- $\alpha$ forest with $N_{\mathrm{HI}}$ between $10^{14}$ and $10^{16} \mathrm{~cm}^{-2}$, but the medium is strongly photoionized, with the neutral fraction around $10^{-6}-10^{-5}$ (the total column density is thus much higher). Typical sizes are $200 \mathrm{kpc}$, certainly 
in filamentary structures. Recent ionized helium detections (Jacobsen et al. 1994, Davidsen et al. 1996) reveal that the most diffuse regions of the intergalactic medium are filled with ionized gas, in other words the GunnPeterson effect is detected in HeII.

What is less well known is the high end of the density spectrum, and whether small clumps of dense molecular gas exist in the intergalactic medium. In the frame of the fractal model or hierarchy of gas structures proposed for the ISM, physical mechanisms can explain the dynamical equilibrium and the non star formation (Pfenniger \& Combes 1994). These structures, down to the smallest $\mathrm{H}_{2}$ clumps, could be formed at high redshift $(z>100)$, as soon as the cooling time is much smaller than the Hubble time. The clumps will form before re-heating, and represent an alternative model to solve the cooling catastrophe.

In any case, galaxies are not isolated objects, they are connected to the gas reservoirs of the intergalactic medium, which contains most of the baryons at $z=2$. This should be taken into account when considering galaxy interactions. If the external gas has a flattened geometry, a big disk or ring can form in a merging sequence (as observed in NGC 520 for instance). The interaction produces compression, higher densities and higher neutral fraction (which goes as the square of the density) and the gas will become visible in the HI line.

\section{Conclusion}

There are various pieces of evidence for large gas reservoirs in the outer parts of galaxies. This gas is driven inwards during galaxy interactions, through the associated gravity torques. It can help to explain mergers and the triggered starbursts, permanent gas accretion in warps, polar rings, etc. If the medium is clumpy and fractal, the gas mass could be underestimated by factors $2-10$, and this can have important cosmological consequences.

\section{References}

Adler D.S., Allen R.J., Lo K.Y.: 1991, ApJ 382, 475

Becquaert J-F., Combes F.: 1997, A\&A 345, 41

Bosma A.: 1981, AJ 86, 1971

Braine J., Combes F.: 1993, A\&A 269, 7

Broeils A.: 1992, PhD Thesis, Groningen

Broeils A., van Woerden H.: 1994, A\&AS 107, 129

Broeils A., Rhee M.H.: 1997, A\&A 324, 877

Burton W.B.: 1992, in "The Galactic Interstellar Medium", Saas-Fee Advanced Course 21, ed. D. Pfenniger \& P. Bartholdi, p. 1

Casoli F., Dickey J., Kazes I. et al.: 1996, A\&A 309, 43

Cayatte V., Balkowski C., van Gorkom J.H., Kotanyi C.: 1990, AJ 100, 604

Cayatte V., Kotanyi C., Balkowski C., van Gorkom J.H.: 1994, AJ 107, 1003

Combes F., Pfenniger D.: 1997, A\&A 327, 453 
Corbelli E., Salpeter E.E.: 1993, ApJ 419, 94 \& 104

Corbelli E., Schneider S.E., Salpeter E.E.: 1989, AJ 97, 390

Davidsen A.F., Kriss G.A., Zheng W.: 1996, Nature 380, 47

Freeman K.C.: 1993, in "Physics of nearby galaxies, Nature or Nurture?" ed. T.X. Thuan, C. Balkowski, Van J.T.T., Editions Frontières, Gif-sur-Yvette, p. 201

Guélin M., Zylka R., Mezger P.G. et al.: 1993, A\&A 279, L37

Hibbard J.E.: 1995, PhD thesis, Columbia University

Hoffman G.L., Salpeter E.E., Farhat B. et al.: 1996, ApJS 105, 269

Huchtmeier W.K., Seiradakis J.H.: 1985, A\&A 143, 216

Jacobsen P. et al.: 1994, Nature 370, 35

Kamphuis J., Briggs F.: 1992, A\&A 253, 335

Madau P. 1992, ApJ 389, L1

Moore B., Katz N., Lake G.: 1996, ApJ 457, 455

Nelson A.E., Zaritsky D., Cutri R.M.: 1996, AAS, 189, 6706

Neininger N., Guélin M., Garcia-Burillo S., Zylka R., Wielebinski R.: 1996, A\&A 310, 725

Olling R.P.: 1995, AJ 110, 591

Olling R.P.: 1996, AJ 112, 457

Pfenniger D., Combes F., Martinet L.: 1994, A\&A 285, 79

Pfenniger D., Combes F.: 1994, A\&A 285, 94

Renzini A.: 1997, ApJ 488, 35

Richter O-G., Sancisi R.: 1994, A\&A 290, L9

Smartt S.J., Rolleston W.R.: 1997, ApJ 481, L47

Scoville, N.Z., Sargent, A.I., Sanders, D.B., Soifer, B.T.: 1991 ApJ, 366, L5

Solomon P.M., Downes D., Radford S.J.E., Barrett J.W.: 1997, ApJ 478, 144

van Gorkom J.H.: 1991, in "Atoms, Ions and Molecules", ed. A.D. Haschik, ASP Conf. Ser. 16, 1

Yun M.S., Ho, P.T.P., Lo K.Y.: 1993, ApJ, 411, L17 